\title{
Regularity and identification of Generalized Multifractional Gaussian Processes
}

Ayache Antoine $^{1}$, Benassi Albert ${ }^{2}$, Cohen Serge ${ }^{3}$, and Lévy Véhel Jacques ${ }^{4}$

${ }^{1}$ Université Paul Sabatier

UFR MIG, Laboratoire de Statistiques et de Probabilités 118, Route de Narbonne, 31062 Toulouse, France

ayache@math . ups-tlse.fr

${ }^{2}$ Université Blaise Pascal (Clermont-Ferrand II), LaMP, CNRS UPRESA 6016, 63177 Aubière Cedex, France

benassi@opgc. univ-bpclermont.fr

${ }^{3}$ Université Paul Sabatier

UFR MIG, Laboratoire de Statistiques et de Probabilités

118, Route de Narbonne, 31062 Toulouse, France

scohen@cict.fr

${ }^{4}$ INRIA-Groupe Fractales,

B.P. 105 Domaine de Voluceau

78153 Le Chesnay Cedex, France

Jacques.Levy_Vehel@inria.fr

Summary. In this article a class of multifractional processes is introduced, called Generalized Multifractional Gaussian Process (GPMP). For such multifractional models, the Hurst exponent of the celebrated Fractional Brownian Motion is replaced by a function, called the multifractional function, which may be irregular. The main aim of this paper is to show how to identify irregular multifractional functions in the setting of GMGP. Examples of discontinuous multifractional functions are also given.

Key words: Gaussian processes, identification, multifractional function. AMS classification (2000): 60G15, 62G05.

\section{Introduction}

Fractional Brownian Motion, that was introduced in [16] and studied in [19], is the continuous mean-zero Gaussian process $\left\{B_{H}(t)\right\}_{t \in \mathbb{R}}$, depending on a parameter $H \in(0,1)$, called the Hurst index, with covariance kernel

$$
\mathbb{E}\left(B_{H}(s) B_{H}(t)\right)=c\left(|s|^{2 H}+|t|^{2 H}-|s-t|^{2 H}\right),
$$

where $s, t$ are arbitrary reals and $c>0$ is a constant. This process has important applications in modeling [6]. One of its main interests is that its pointwise Hölder regularity can be prescribed via its Hurst parameter. Indeed, 
the Hölder exponent of FBM, at any point, is equal to $H$, almost surely. Recall that, for a stochastic process $X$, the Hölder exponent at a point $t_{0}$, is defined as

$$
\alpha_{X}\left(t_{0}\right)=\sup \left\{\alpha, \limsup _{h \rightarrow 0} \frac{\left|X\left(t_{0}+h\right)-X\left(t_{0}\right)\right|}{|h|^{\alpha}}=0\right\} .
$$

However, the Hölder exponent of FBM remains the same all along its trajectory and this can be restrictive in some situations. For this reason, various models have been proposed to replace the Hurst index $H \in(0,1)$ by a function $h(t)$. These so-called multifractional models ${ }^{1}$ are useful in various domains of applications, see for instance $[1,15,18,11,17]$. The starting point of these generalizations is very often the harmonizable representation of the FBM :

$$
B_{H}(t)=\int_{\mathbb{R}} \frac{e^{-i t \cdot \xi}-1}{|\xi|^{\frac{1}{2}+H}} W(d \xi)
$$

where $W(d \xi)$ is a Wiener measure such that $X$ is a real valued process (cf. [12] or page 1138 in [1] for a discussion on the Wiener measure). For example we may recall the Multifractional Brownian Motion (MBM), whose harmonizable representation is given by :

$$
B_{h}(t)=\int_{\mathbb{R}} \frac{e^{-i t \cdot \xi}-1}{|\xi|^{\frac{1}{2}+h(t)}} W(d \xi)
$$

where $h$ is a Hölder function ranging in $[a, b] \subset(0,1)$. The function $h$ is called the multifractional function. This process has been introduced independently in [22] (where the denomination MBM was introduced) and in [10]. Similarly to FBM, MBM is continuous and its Hölder exponent at any point $t_{0}$ is equal to $h\left(t_{0}\right)$, almost surely. However, one of the main problems with these models is that their Hölder exponents cannot be irregular, because $h$ must be Hölderian. Recall that when the function $h$ is discontinuous then the trajectories of the MBM are themselves, with probability 1, discontinuous (see Proposition 1 of [1]).

The continuity of $h$ is a real drawback for some applications, for instance image classification and segmentation: The Hölder regularity is expected to vary abruptly on textured zones and around edges. On the other hand, the identification of $h$ has been performed under the assumption that $h$ is continuously differentiable [8].

Recently, two continuous Gaussian processes with irregular Hölder exponents have been introduced. These processes are the Generalized Multifractional Brownian Motion (in short GMBM) [5, 4] and the Step Fractional

\footnotetext{
${ }^{1}$ One should not confuse multifractional and multifractal. A multifractal process has a multifractal spectrum that takes non trivial values on a set of positive measure. A multifractional process is a process with non constant pointwise Hölder exponent. Multifractality implies multifractionality, while the converse is false.
} 
Brownian Motion (in short SFBM) [7]. The GMBM is an extension of the MBM while the SFBM is an extension of the FBM. In this article we will study a Gaussian process that is slightly more general than the GMBM, so let us first recall the definition of the GMBM. Let $\lambda>1$ be an arbitrary fixed real, and $(h)=\left(h_{n}\right)_{n \in \mathbb{N}}$ be a sequence of Hölder functions with values in $[a, b] \subset(0,1)$. The GMBM with parameters $\lambda$ and $(h)$ is the continuous Gaussian process $\left\{Y_{(h)}(t)\right\}_{t \in \mathbb{R}}$ defined for every real $t$ as,

$$
\begin{aligned}
Y_{(h)}(t) & =\int_{0<\xi<1} \frac{e^{-i t \cdot \xi}-1}{\xi^{\frac{1}{2}+h_{0}(t)}} W(d \xi) \\
& +\sum_{n=1}^{+\infty} \int_{\lambda^{n-1} \leq \xi<\lambda^{n}} \frac{e^{-i t \cdot \xi}-1}{\xi^{\frac{1}{2}+h_{n}(t)}} W(d \xi) .
\end{aligned}
$$

One of the main properties of the GMBM is that its Hölder exponent at any point $t_{0}$ is almost surely equal to $\liminf _{n \rightarrow \infty} h_{n}\left(t_{0}\right)$, under the assumption that $\left\|h_{n} \mid\right\|=o\left(2^{n a}\right)$, where $\left\|\left|h_{n}\right|\right\|$ denotes the Hölder norm of $h_{n}$ (see $[2,5,4]$ ). Therefore, the Hölder exponent of the GMBM is of the most general form : any liminf of positive continuous functions (see $[14,3,2]$ ). In contrast, the one of SFBM is a step function (see subsection 1.3.1 in [7]). As a consequence, the Hölder exponent of the GMBM may be discontinuous everywhere while that of the SFBM can only be discontinuous on a finite set. Likewise, the Hölder exponent of the GMBM may be constant except on a finite set (see Proposition 1 of [5]), while this is impossible with the SFBM. On the other hand, the parameters of the SFBM have been identified in [7], and we present in this article an identifiable model which has many desirable properties of the GMBM.

Let us define a Generalized Multifractional Gaussian Process (in short GMGP) by its harmonizable representation :

$$
X(t)=\int_{0}^{+\infty} \frac{e^{-i t \cdot \xi}-1}{\xi^{\frac{1}{2}+H(t, \xi)}} W(d \xi) .
$$

where $W(d \xi)$ is a Wiener measure such that $X$ is real valued process and $H$ : $\mathbb{R} \times(0,+\infty) \rightarrow[a, b] \subset(0,1)$ is called the Hurst function. Clearly the MBM is a GMGP for which $H(t, \xi)$ does not depend on the frequency variable $\xi$ and is always equal to the multifractional function $h(t)$. Actually the GMGP model is very close to the GMBM: On the one hand, every GMBM with parameters $\lambda$ and $\left(h_{n}\right)_{n \in \mathbb{N}}$ is a GMGP with Hurst function $H(t, \xi)=\mathbf{1}_{[0,1)}(\xi) h_{0}(t)+$ $\sum_{n=1}^{+\infty} \mathbf{1}_{\left[\lambda^{n-1}, \lambda^{n}\right)}(\xi) h_{n}(t)$. On the other hand, if $H: \mathbb{R} \times(0,+\infty) \rightarrow[a, b] \subset$ $(0,1)$ is continuous in almost all $\xi$, then it is not hard to see that the high frequencies part of the GMBM of parameter $\lambda$ and $\left(H\left(., \lambda^{n}\right)\right)_{n \in \mathbb{N}}$ converges, in distribution to that of the GMGP with Hurst function $H(.,$.$) , when \lambda \rightarrow 1$. As for the SFBM, it is also, in some sense, similar to the GMGP, although, 
contrarily to the SFBM, no wavelet expansion is needed to introduce the GMGP. We refer to [13] for a comparison between SFBM and GMBM with parameter $\lambda=2$.

The remaining of this paper is organized as follows. In section 2 , we first obtain the pointwise Hölder exponent of the GMGP. We then define our estimator and we give the precise assumptions ensuring its almost sure convergence. Section 3 is devoted to three examples where the Hurst functions $H(t, \xi)$ converge to multifractional functions of particular interest. The proof of the results are performed in section 4 .

\section{Statement of the results}

Following the correspondence between the GMBM and the GMGP, the multifractional function of a GMGP is defined to be :

$$
h(t)=\lim _{\xi \rightarrow+\infty} H(t, \xi),
$$

when the limit exists. This definition is coherent with the fact that the local properties of the sample paths are given by the high frequencies of the harmonizable representation. In this paper we will show that, similarly to the GMBM, at any point $t_{0}$, the Hölder exponent of the GMGP is almost surely equal to $h\left(t_{0}\right)$. To identify the multifractional function that governs the regularity of the sample paths, we will then use the generalized quadratic variation

$$
V_{N}=\sum_{p=0}^{N-2}\left(X\left(\frac{p+2}{N}\right)-2 X\left(\frac{p+1}{N}\right)+X\left(\frac{p}{N}\right)\right)^{2} .
$$

Actually first the infimum of the multifractional function $\inf _{t \in(0,1)} h(t)=h_{*}$ will be identified. Before stating the main results and making precise the technical assumptions we need to explain why $h_{*}$ is naturally identified in this framework. As a rule of the thumb one may assume for multifractional models that

$$
\mathbb{E} V_{N} \approx \sum_{p=0}^{N-2} \frac{1}{N^{2 h(p / N)}}
$$

and then it is classical to have

$$
\lim _{N \rightarrow+\infty} \frac{\ln \left(\mathbb{E} V_{N}\right)}{\ln (N)}=1-2 h_{*} .
$$

In this article we will prove a Law of the Large Numbers for $V_{N}$ and the preceding heuristic suggests to take as an estimator :

$$
\widehat{h}_{N}=1 / 2\left(1-\frac{\ln \left(V_{N}\right)}{\ln (N)}\right) .
$$


Let us now state more precisely the technical assumptions needed to determine the Hölder regularity of GMGP and to show that $\widehat{h}_{N}$ is a consistent estimator of $h_{*}$. First of all we state a weak assumption on the multifractional function $h$ that will entail (9) :

A $1 \exists C>0, \forall N \geq 1$

$$
\frac{1}{C} N^{1-2 h_{*}} \leq \sum_{p=0}^{N-2} \frac{1}{N^{2 h(p / N)}} \leq C N^{1-2 h_{*}} .
$$

Note that $C$ is a generic constant that may change in this article and that the last inequality of (11) is always satisfied. The lower bound in (11) means that there is a sufficiently large number of points $p / N$ such that $h(p / N)$ is close to $h_{*}$.

The asymptotic behavior (11) is clearly true under mild assumptions of smoothness for $t \rightarrow h(t)$ and we will see in the examples that it is fulfilled for non trivial functions $h(t)$.

One needs then to strengthen the existence of the limit (6) to ensure that (8) is satisfied. The following technical assumption states that the speed of convergence of $H(t, \xi)$ to $h(t)$ is compatible with (8).

A $2 \forall 0 \leq t \leq 1, \quad \forall \xi \geq 0, \quad H(t, \xi) \geq h(t)$. $\exists \alpha<1$ such that

$$
\sum_{p=0}^{N-2} \int_{N^{\alpha}}^{+\infty} \frac{|h(p / N)-H(p / N, \xi)|}{\xi^{2 h(p / N)+1}} \ln (\xi) d \xi=o\left(N^{1-2 h_{*}}\right) .
$$

Let us make some comments to explain (12). In our setting, it will yield that $\mathbb{E} V_{N}$ is sufficiently close to $N^{1-2 h_{*}}$ to have (9). Now why is (12) a reasonable assumption? Let us suppose that $|H(p / N)-H(p / N, \xi)| \leq C_{N}$. Then:

$$
\left|\sum_{p=0}^{N-2} \int_{N^{\alpha}}^{+\infty} \frac{|h(p / N)-H(p / N, \xi)|}{\xi^{2 h(p / N)+1}} \ln (\xi) d \xi\right| \leq \sum_{p=0}^{N-2} \frac{C_{N} \ln N}{N^{2 \alpha h(p / N)}} .
$$

If we assume that $\lim _{N \rightarrow+\infty} C_{N} \ln N N^{2 \alpha b}=0$, then (12) is satisfied because of A 1. Hence $\mathbf{A} \mathbf{2}$ can be interpreted as a way of expressing that $H(t, \xi)$ is converging uniformly fast enough to $h(t)$ when $\xi \rightarrow+\infty$.

The last assumption is concerned with the behaviour of the functions $H(., \xi)$ when $\xi$ is finite. It expresses the fact that these functions must be smooth enough so that the Hölder regularity of the GMGP is indeed governed by the multifractional function.

A 3 - For $0<\xi \leq \frac{4 \pi}{3}$,

$$
H(t, \xi)=b
$$


- for $\frac{4 \pi}{3}<\xi \exists g$ such that $0<g<\min (a / 2,1 / 4)$ (where $\left.H(t, \xi) \in[a, b]\right)$ and $\exists \beta$ such that $b<\beta \leq 1$

$$
\left|\frac{\partial^{k} H}{\partial \xi^{k}}(t, \xi)-\frac{\partial^{k} H}{\partial \xi^{k}}\left(t^{\prime}, \xi\right)\right| \leq C|\xi|^{g-k}\left|t-t^{\prime}\right|^{\beta}
$$

for $t, t^{\prime} \in[0,1]$ and $k=0,1,2$. Moreover

$$
\left|\frac{\partial^{k} H}{\partial \xi^{k}}(t, \xi)\right| \leq C \xi^{g-k}
$$

for $t \in[0,1]$ and $k=0,1,2$.

Of course, in these assumptions, $\frac{4 \pi}{3}$ is arbitrary and can be replaced by any non-negative constant. The inequality $0<g<\min (a / 2,1 / 4)$ has a deeper meaning : it expresses the fact that the Hölder constant of $H(t, \xi)$ cannot grow too fast when $\xi \rightarrow+\infty$. Finally, the inequality $b<\beta$ is similar to the condition $r>\sup (a(x))$ in the Definition $1.4 \mathrm{p} 41$ of [10] or to the condition $\sup _{t} H(t)<\beta$ in Definition $3.1 \mathrm{p} 11$ of [4] ; we refer to [13] section 4.2 or to [1] section 2.2 for a more complete discussion.

At this point, we can state the three main results of this paper.

Theorem 1. Under assumption A 3, the Hölder exponent of the GMGP at each point $t_{0}$ satisfies almost surely,

$$
\alpha_{X}\left(t_{0}\right)=h\left(t_{0}\right) \text {. }
$$

Theorem 2. Under assumptions A 1, A 2, A 3,

$$
\lim _{N \rightarrow+\infty} \widehat{h}_{N}=h_{*} \quad \text { almost surely. }
$$

Actually, the identification of the infimum $h_{*}$ of the multifractional function $h$ is the result needed to obtain the identification of $h$ itself. The classical technique to have full identification is the localization of the generalized quadratic variations. Basically if one wants to estimate $h(t)$ for a given $t \in(0,1)$, one has first to localize $V_{N}$ on an $(\varepsilon, N)$-neighborhood of $t$ defined by

$$
\mathcal{V}_{\varepsilon, N}(t)=\left\{p \in \mathbb{Z},\left|\frac{p}{N}-t\right| \leq \varepsilon\right\} .
$$

Then the localized generalized variation is defined by

$$
\mathbf{V}_{\varepsilon, N}(t)=\sum_{p \in \mathcal{V}_{\varepsilon, N}(t)}\left(X\left(\frac{p+1}{N}\right)-2 X\left(\frac{p}{N}\right)+X\left(\frac{p-1}{N}\right)\right)^{2}
$$

We define the localized estimator

$$
\widehat{h}_{\varepsilon, N}=1 / 2\left(1-\frac{\ln \left(\mathbf{V}_{\varepsilon, N}(t)\right)}{\ln (N)}\right),
$$


and we get

$$
\lim _{N \rightarrow+\infty} \widehat{h}_{\varepsilon, N}=\inf _{\{|s-t|<\varepsilon\}} h(s) \quad \text { almost surely }
$$

as in Remark 1 in [8].

The next step to identify $h$ at point $t$ is to let $\varepsilon \rightarrow 0^{+}$. For instance, taking $\varepsilon=N^{-\gamma}$ for $\gamma>0$, one obtains the following result as a corollary of Theorem 2:

Corollary 1. Fix $0<\gamma<1 / 2$. Under assumptions A 1, A 2 and A 3,

$$
\lim _{N \rightarrow+\infty} 1 / 2\left(1-\gamma-\frac{\ln \left(\boldsymbol{V}_{N^{-\gamma, N}}(t)\right)}{\ln (N)}\right)=\liminf _{s \rightarrow t} h(s) \quad \text { a.s. }
$$

The statement of this corollary is an example of localization of Theorem 2 . Remark that if $h$ is lower semi-continuous and satisfies A 1, A 2, and A 3, then it is identifiable.

\section{Examples}

In this section we present three examples of application of Theorem 2, that illustrate the way this theorem can be used. In each of these examples, one assumes given a precise multifractional function. Then, one constructs a GMGP by exhibiting a Hurst function $H(t, \xi)$ such that the assumptions A 1, A 2 and $\mathbf{A} \mathbf{3}$ are fulfilled. Techniques similar to the ones used in this section were put to work in the proofs of Propositions 1 and 2 of [5].

\subsection{Hölder continuous multifractional functions}

Remark first that if the multifractional function $h$ is $\beta$-Hölder continuous with $\beta>b$ and satisfies $\mathbf{A} \mathbf{1}$, one may choose a Hurst function $H(t, \xi)$ that interpolates a constant for $0 \leq \xi \leq \frac{4 \pi}{3}$ and the multifractional function $h(t)$ when $\xi \geq \frac{8 \pi}{3}$. One can take for instance

$$
H(t, \xi)=b \quad \text { if } \quad 0 \leq \xi \leq \frac{4 \pi}{3}
$$

and

$$
H(t, \xi)=\left(2-\frac{3 \xi}{4 \pi}\right) b+\left(\frac{3 \xi}{4 \pi}-1\right) h(t) \quad \text { if } \quad \frac{4 \pi}{3} \leq \xi \leq \frac{8 \pi}{3},
$$

and $H(t, \xi)=h(t)$ if $\xi \geq \frac{8 \pi}{3}$. In this case the GMGP exhibits the same properties as a Multifractional Brownian Motion and the Theorem 2 is the analogue of the Remark 1 in [8] where the identification of a multifractional function is performed by localizing the generalized quadratic variations as in [8]. Please note that an alternate method to corollary 1 has recently been proposed in [21] to identify the multifractional function of a MBM in the Hölderian case. 


\subsection{Multifractional functions with one jump}

In this example we consider a multifractional function that has only one discontinuity and that is piecewise constant. This very simple example can be easily extended to the case of piecewise constant functions with a finite number of jumps. The GMGP associated to this kind of multifractional function are comparable to SFBM for the applications. Let us suppose that

$$
h(t)=b \mathbf{1}_{\left[0, t_{0}\right]}+a \mathbf{1}_{\left(t_{0}, 1\right]}
$$

where $t_{0} \in(0,1)$ and $0<a<b<1$. A 1 is clearly satisfied with $h_{*}=a$. Then one can construct a Hurst function that admits such a multifractional function through the following procedure :

- For $0<\xi \leq \frac{4 \pi}{3}$,

$$
H(t, \xi)=b \quad \forall t \in[0,1] .
$$

- for $\frac{4 \pi}{3}<\xi$ let us choose $0<f<\min (a / 6,1 / 12)$

$$
\begin{aligned}
& H(t, \xi)=b \\
& =a+(b-a) \sin ^{4}\left(\frac{\pi \xi^{f}}{2}\left(t-t_{0}-\frac{1}{\xi^{f}}\right)\right) \quad \text { if } \quad t \in\left[t_{0}, t_{0}+\frac{1}{\xi^{f}}\right) \\
& =a \\
& \text { if } t \geq t_{0}+\frac{1}{\xi^{f}} \text {. }
\end{aligned}
$$

An elementary computation shows that $\mathbf{A} \mathbf{3}$ is fulfilled with $g=3 f$ and $\beta=1$. To check $\mathbf{A} \mathbf{2}$, let us remark that, if $\sharp$ denotes the cardinality of a set,

$$
\begin{aligned}
& \left|\sum_{p=0}^{N-2} \int_{N^{\alpha}}^{+\infty} \frac{|h(p / N)-H(p / N, \xi)|}{\xi^{2 h(p / N)+1}} \ln (\xi) d \xi\right| \leq \\
& \quad(b-a) \sharp\left\{p \text { such that } t_{0} \leq p / N \leq t_{0}+\frac{1}{N^{\alpha f}}\right\} \times \int_{N^{\alpha}}^{+\infty} \frac{\ln (\xi)}{\xi^{2 a+1}} d \xi .
\end{aligned}
$$

Hence

$$
\left|\sum_{p=0}^{N-2} \int_{N^{\alpha}}^{+\infty} \frac{|h(p / N)-H(p / N, \xi)|}{\xi^{2 h(p / N)+1}} \ln (\xi) d \xi\right| \leq \frac{C N \ln N}{N^{\alpha f}} \frac{1}{N^{2 h_{*} \alpha}} .
$$

Then $\alpha$ is chosen such that $\alpha\left(f+2 h_{*}\right)>2 h_{*}$ to have (12).

\subsection{Multifractional functions with accumulation of jumps}

Let us address the more interesting case of piecewise constant multifractional functions which have an infinite number of jumps. In this example let us suppose the multifractional function is defined by 


$$
\begin{array}{rlrl}
h(t) & =1 / 2 & \text { if } t & \leq 1 / 2 \\
& =\frac{1}{2}+\frac{1}{k+1} & \text { if } \frac{1}{2}+\frac{1}{k+1} & <t \leq \frac{1}{2}+\frac{1}{k} \\
& =\frac{1}{2}+\frac{1}{3} & \text { if } 1 \leq t
\end{array}
$$

where $k \geq 2$. This multifractional function fulfills $\mathbf{A} 1$ with $h_{*}=1 / 2$, and $t=1 / 2$ is the time where the jumps accumulate. Let us now exhibit a Hurst function corresponding to (21) by using the construction of the previous example which is valid when the multifractional function has only a finite number of jumps. Let us define the function (21) where only $k_{0}$ jumps are considered :

$$
\begin{aligned}
h_{k_{0}}(t) & =1 / 2 & \text { if } t & \leq 1 / 2 \\
& =\frac{1}{2}+\frac{1}{k+1} & & \text { if } \frac{1}{2}+\frac{1}{k+1}<t \leq \frac{1}{2}+\frac{1}{k}, \text { for } k \leq k_{0} \\
& =\frac{1}{2}+\frac{1}{k_{0}+1} & & \text { if } \frac{1}{2}+\frac{1}{k_{0}} \leq t .
\end{aligned}
$$

Then one can construct, with the same technique as in the example of section 3.2 , a Hurst function corresponding to $h_{k_{0}}$ which is denoted by $H_{k_{0}}(t, \xi)$ and is defined for $\left(k_{0}\left(k_{0}+1\right)\right)^{1 / f} \leq \xi$. If we fix $f<\min (a / 6,1 / 12)$ in the previous example, then this last condition simply means that $\left[\frac{1}{2}+\frac{1}{k}, \frac{1}{2}+\frac{1}{k}+\frac{1}{\xi^{f}}\right]$ is included in the intervals where $h_{k_{0}}$ is constant. Hence this condition allows us to manage the jumps of $h_{k_{0}}$ separately. Let us now introduce a Hurst function corresponding to (21) by taking more and more jumps into account when $\xi \rightarrow+\infty$. Define

$$
K(\xi)=\sup \left\{k \in \mathbb{N}^{*} \text { such that }(k(k+1))^{1 / f} \leq \xi\right\}
$$

and define the Hurst function with the $H_{k_{0}}$ 's

$$
H(t, \xi)=H_{K(\xi)}(t, \xi) .
$$

It is not difficult to check that this Hurst function satisfies $\mathbf{A} \mathbf{3}$ since

$$
\frac{\partial^{m} H}{\partial \xi^{m}}(t, \xi)=\frac{\partial^{m} H_{k_{0}}}{\partial \xi^{m}}(t, \xi)
$$

when $\left(k_{0}\left(k_{0}+1\right)\right)^{1 / f} \leq \xi \leq\left(\left(k_{0}+1\right)\left(k_{0}+2\right)\right)^{1 / f}$. To check A 2 let us write

$$
\int_{N^{\alpha}}^{+\infty} \sum_{p=0}^{N-2} \frac{|h(p / N)-H(p / N, \xi)|}{\xi^{2 h(p / N)+1}} \ln (\xi) d \xi=I+J
$$

where

$$
I=\int_{N^{\alpha}}^{+\infty} \sum_{p=0}^{\left[\frac{N}{2}+\frac{N}{K(\xi)+1}\right]} \frac{\frac{1}{K(\xi)+1}}{\xi^{2 h(p / N)+1}} \ln (\xi) d \xi
$$


and where $[x]$ stands for the integral part of $x .|h(p / N)-H(p / N, \xi)|$ has been replaced by its constant value for the considered $p^{\prime} s$. The second part is

$$
J=\int_{N^{\alpha}}^{+\infty} \sum_{p=\left[\frac{N}{2}+\frac{N}{K(\xi)+1}\right]+1}^{N-2} \frac{\left|h_{K(\xi)}(p / N)-H_{K(\xi)}(p / N, \xi)\right|}{\xi^{2 h(p / N)+1}} \ln (\xi) d \xi .
$$

Let us upper-bound $I$ by remarking that if $N^{\alpha} \leq \xi$ then

$$
\frac{1}{K(\xi)+1} \leq C N^{\frac{-\alpha f}{2}} \text {. }
$$

Hence

$$
|I| \leq N \times C N^{\frac{-\alpha f}{2}} \int_{N^{\alpha}}^{+\infty} \frac{\ln (\xi)}{\xi^{2}} d \xi
$$

and $\alpha$ is chosen such that

$$
|I| \leq C \frac{N \ln (N)}{N^{\alpha(1+f / 2)}}=o(1)
$$

which is the required estimate since $h_{*}=1 / 2$.

To upper-bound $J$ let us remark that for a fixed $\xi, h_{K(\xi)}$ has $K(\xi)$ jumps at time $\frac{1}{2}+\frac{1}{k+1}$ of magnitude $\frac{1}{k}-\frac{1}{k+1}$. Hence

$$
\begin{aligned}
\sum_{p=\left[\frac{N}{2}+\frac{N}{K(\xi)+1}\right]+1}^{N-2}\left|h_{K(\xi)}(p / N)-H_{K}(\xi)(p / N, \xi)\right| & \leq C N^{1-\alpha f} \sum_{k=1}^{K(\xi)}\left(\frac{1}{k}-\frac{1}{k+1}\right) \\
& \leq C N^{1-\alpha f}
\end{aligned}
$$

where the factor $C N^{1-\alpha f}$ comes from $\sharp\left\{p\right.$ such that $\left.t_{0} \leq p / N \leq t_{0}+\frac{1}{N^{\alpha f}}\right\}$ for each jump $t_{0}$ as in the previous example. Then

$$
|J| \leq C N^{1-\alpha f} \frac{\ln (N)}{N^{\alpha}}
$$

which yields (12) for a convenient $\alpha$.

\section{Proof of the results}

In this section, Theorems 1, 2 and Corollary 1 are proved. First let us describe the main steps of the proofs. In the definition (5) of the GMGP we remark that the integrand

$$
\frac{e^{-i t \cdot \xi}-1}{|\xi|^{\frac{1}{2}+H(t, \xi)}}
$$

depends of the variable $t$ twice. To study the generalized variation $V_{N}$ a new process is considered 


$$
Y(s, t)=\int_{0}^{+\infty} \frac{e^{-i s \cdot \xi}-1}{|\xi|^{\frac{1}{2}+H(t, \xi)}} W(d \xi)
$$

where this dependence is split into two variables $(s, t)$. Then a variation $W_{N}$ associated to this process $Y$ is introduced

$$
W_{N}=\sum_{p=0}^{N-2}\left(Y\left(\frac{p+2}{N}, \frac{p}{N}\right)-2 Y\left(\frac{p+1}{N}, \frac{p}{N}\right)+Y\left(\frac{p}{N}, \frac{p}{N}\right)\right)^{2}
$$

$W_{N}$ is simpler to study than $V_{N}$ because its variation does not involve the variable $t$ that appears in the Hurst function. Moreover one can see easily that

$$
V_{N}=\sum_{p=0}^{N-2}\left(Y\left(\frac{p+2}{N}, \frac{p+2}{N}\right)-2 Y\left(\frac{p+1}{N}, \frac{p+1}{N}\right)+Y\left(\frac{p}{N}, \frac{p}{N}\right)\right)^{2} .
$$

A result very similar to Lemma 3.6 in [2] (namely Proposition 1 below) shows that the process $Y$ is Hölder continuous in the second variable uniformly with respect to the first variable. This fact yields the regularity result of Theorem 1 and that $V_{N}$ can be replaced by $W_{N}$. This is the first step in the proof of Theorem 2. Then it is shown that

$$
\lim _{N \rightarrow+\infty} \frac{\ln \left(\mathbb{E} W_{N}\right)}{\ln (N)}=1-2 h_{*}
$$

in the second step. The third step consists in a study of the asymptotic of the variance of $W_{N}$ that allows to replace $\mathbb{E} W_{N}$ by $W_{N}$ in the previous limit. Finally Corollary 1 is proved.

\subsection{Study of $Y$.}

In this part we prove the Hölder continuity of $Y$. Although the main lines of the proof of the following Proposition are similar to those of Lemma 3.6 in [2], we will give this proof for completeness.

Proposition 1. Under assumption A 3, there exists an almost surely positive random variable $C(\omega)$ such that :

$$
\sup _{s \in[0,1]}\left|Y(s, t)-Y\left(s, t^{\prime}\right)\right| \leq C(\omega)\left|t-t^{\prime}\right|^{\beta}
$$

for $t, t^{\prime} \in[0,1]$ where $\beta$ is defined in (13).

Proof : To get (28) we will apply a series expansion of $Y$ in the orthonormal basis of $L^{2}$ given by the Lemarié Meyer wavelets (cf. [20]). This technique is classical to study the smoothness of multifractional process. As in [2] for the GMBM (see Relation (3.7)) or in [10] for the MBM we get 


$$
Y(s, t)=\sum_{j=0}^{+\infty} \sum_{k=-\infty}^{+\infty} s_{j, k}(s, t) \eta_{j, k}
$$

where $\eta_{j, k}$ are identically distributed standard Gaussian variables and where the coefficients are :

$$
s_{j, k}(s, t)=2^{-j / 2} \int_{0}^{+\infty} \frac{e^{-i s \cdot \xi}-1}{\xi^{\frac{1}{2}+H(t, \xi)}} \overline{\psi_{j, k}}(\xi) d \xi .
$$

Please note that equation (29) is only true up to an additive smooth process. In (30) $\left(\psi_{j, k}\right)_{j, k \in \Lambda^{+}}$is the Lemarié Meyer basis indexed by $\Lambda^{+}=\{j, k, \quad j \geq$ 0 , and $\quad k \in \mathbb{Z}\}$ as explained in [20] or in page 29 of [10]. Then the Hölder continuity of $Y$ derives from estimates on the coefficients $s_{j, k} \quad[2]$

$\exists \epsilon, \forall k \in \mathbb{Z}$

$$
\left|s_{j, k}(s, t)\right| \leq C 2^{-j \epsilon}\left\{\frac{1}{\left(1+\left|2^{j} s-k\right|^{2}\right)}+\frac{1}{1+k^{2}}\right\},
$$

for $j \geq 1$

$$
\left|s_{j, k}(s, t)-s_{j, k}\left(s, t^{\prime}\right)\right| \leq C j 2^{-j \epsilon}\left|t-t^{\prime}\right|^{\beta}\left\{\frac{1}{\left(1+\left|2^{j} s-k\right|^{2}\right)}+\frac{1}{1+k^{2}}\right\} .
$$

Note that the estimates (31) and (32) are quite similar to those in Lemma 3.3 in [2]. This Lemma is, to a certain extent, inspired from Proposition 4.1, page 79 in [10]. The estimate (31) and classical almost sure bounds for the supremum of Gaussian variables yield that the series in (29) converges almost surely. Then (32) yields the the Hölder continuity of $Y$. Please remark that

$$
Y(s, t)-Y\left(s, t^{\prime}\right)=\sum_{j=1}^{+\infty} \sum_{k=-\infty}^{+\infty}\left(s_{j, k}(s, t)-s_{j, k}\left(s, t^{\prime}\right)\right) \eta_{j, k}
$$

where the terms for $j=0$ are dropped since the Hurst function is constant on $\left\{\xi \leq \frac{4 \pi}{3}\right\}$. The main ingredient to get these estimates are integrations by parts in the integral of (30). In the framework of GMGP, to carry these integrations by parts, the only new point compared to [2] is the dependence of the Hurst function $H(t, \xi)$ on a continuous parameter $\xi$ instead of a discrete parameter $n$. In the following argument we only stress how the assumption $\mathbf{A} \mathbf{3}$ allows us to control the additional terms caused by this dependence.

Let us study the Hölder continuity of the coefficients $s_{j, k}$ 's. One can write

$$
\begin{aligned}
s_{j, k}(s, t)-s_{j, k}\left(s, t^{\prime}\right)=2^{-j / 2} & \int_{0}^{+\infty}\left(e^{-i s \xi}-1\right) e^{i 2^{-j} k \xi} \\
& \left(\xi^{-\left(\frac{1}{2}+H(t, \xi)\right)}-\xi^{-\left(\frac{1}{2}+H\left(t^{\prime}, \xi\right)\right)}\right) \overline{\widehat{\psi}}\left(2^{-j} \xi\right) d \xi
\end{aligned}
$$


where we have only used the translation-dilatation structure of the Lemarié Meyer basis

$$
\psi_{j, k}(s)=2^{j / 2} \psi\left(2^{j} s-k\right),
$$

for $j \geq 1$. The factor $e^{-i s \xi}-1$ in the previous integral can be split since the support of $\hat{\psi}$ is contained in $\left[\frac{2 \pi}{3}, \frac{8 \pi}{3}\right]$ and the first part leads to

$$
2^{-j / 2} \int_{0}^{+\infty} e^{i\left(k-2^{j} s\right) 2^{-j} \xi}\left(\xi^{-\left(\frac{1}{2}+H(t, \xi)\right)}-\xi^{-\left(\frac{1}{2}+H\left(t^{\prime}, \xi\right)\right)}\right) \overline{\widehat{\psi}}\left(2^{-j} \xi\right) d \xi .
$$

The second part is of the same form but $e^{i\left(k-2^{j} s\right) 2^{-j} \xi}$ is replaced by 1 . Hence this second part yields the second terms in the brackets in the right hand side of (31) and of (32). Let us define

$$
g\left(t, t^{\prime}, \xi\right)=\left(\xi^{-\left(\frac{1}{2}+H(t, \xi)\right)}-\xi^{-\left(\frac{1}{2}+H\left(t^{\prime}, \xi\right)\right)}\right) \overline{\widehat{\psi}}\left(2^{-j} \xi\right) .
$$

After performing two integrations by parts on the last integral one gets for $s \neq 2^{j} k$

$$
2^{-j / 2} \int_{0}^{+\infty} \frac{e^{i\left(k-2^{j} s\right) 2^{-j} \xi}}{i^{2}\left(k-2^{j} s\right)^{2} 2^{-2 j}} \frac{\partial^{2} g\left(t, t^{\prime}, \xi\right)}{\partial \xi^{2}} d \xi
$$

Then the Leibniz rule is applied to the preceding partial derivative

$$
\begin{aligned}
\frac{\partial^{2} g\left(t, t^{\prime}, \xi\right)}{\partial \xi^{2}}=\sum_{p=0}^{2}\left(\begin{array}{l}
2 \\
p
\end{array}\right) \frac{\partial^{p}}{\partial \xi^{p}}\left(\xi^{-\left(\frac{1}{2}+H(t, \xi)\right)}-\xi^{-\left(\frac{1}{2}+H\left(t^{\prime}, \xi\right)\right)}\right) & \\
& 2^{-(2-p) j} \frac{\partial^{2-p} \overline{\widehat{\psi}}}{\partial \xi^{2-p}}\left(2^{-j} \xi\right)
\end{aligned}
$$

Because of the support of $\overline{\widehat{\psi}}$ the partial derivatives of $\bar{\psi}$ can be bounded independently of $j, p$, hence we have only to bound integrals that comes from the factors $\left(\xi^{-\left(\frac{1}{2}+H(t, \xi)\right)}-\xi^{-\left(\frac{1}{2}+H\left(t^{\prime}, \xi\right)\right)}\right)$. For instance for $p=0$

$$
\begin{aligned}
I_{j}=\int_{\frac{2 \pi 2 j}{3}}^{\frac{8 \pi 2^{j}}{3}}\left(\xi^{-\left(\frac{1}{2}+H(t, \xi)\right)}-\xi^{-\left(\frac{1}{2}+H\left(t^{\prime}, \xi\right)\right)}\right) d \xi \\
\quad \leq \int_{\frac{2 \pi 2 j}{3}}^{\frac{8 \pi 2^{j}}{3}}\left|H(t, \xi)-H\left(t^{\prime}, \xi\right)\right| \frac{\ln (\xi)}{\xi^{a+1 / 2}} d \xi
\end{aligned}
$$

Then applying (13) for $k=0$ yields

$$
I_{j} \leq C j 2^{-j\left(a+\frac{1}{2}-g-1\right)}\left|t-t^{\prime}\right|^{\beta}
$$

which is exactly the estimate we need for (32) if we take $0<\epsilon<a-g$. 
Let us now consider the contribution of the term $p=1$ in the sum (33). First

$$
\begin{aligned}
\frac{\partial}{\partial \xi} \xi^{-\left(\frac{1}{2}+H(t, \xi)\right)}=-\frac{1}{2} \xi^{-\left(\frac{3}{2}+H(t, \xi)\right)}-H( & t, \xi) \xi^{-\left(\frac{3}{2}+H(t, \xi)\right)} \\
& -\ln (\xi) \frac{\partial H}{\partial \xi}(t, \xi) \xi^{-\left(\frac{1}{2}+H(t, \xi)\right)} .
\end{aligned}
$$

And the first term of (36) leads to

$$
\left.J_{j, 0}=\frac{-1}{2} \int_{\frac{2 \pi 2^{j}}{3}}^{\frac{8 \pi 2^{j}}{3}}\left(\xi^{-\left(\frac{3}{2}+H(t, \xi)\right)}\right)-\xi^{-\left(\frac{3}{2}+H\left(t^{\prime}, \xi\right)\right)}\right) d \xi \leq C j 2^{j(-\epsilon-1 / 2)}\left|t-t^{\prime}\right|^{\beta} .
$$

This bound gives the same contribution as $I_{j}$ to (32). To conclude the case $p=1$ we have to bound

$$
J_{j, 1}=\int_{\frac{2 \pi 2 j}{3}}^{\frac{8 \pi 2^{j}}{3}}\left(H\left(t^{\prime}, \xi\right) \xi^{-\left(\frac{3}{2}+H\left(t^{\prime}, \xi\right)\right)}-H(t, \xi) \xi^{-\left(\frac{3}{2}+H(t, \xi)\right)}\right) \ln (\xi) d \xi
$$

and

$$
J_{j, 2}=\int_{\frac{2 \pi 2 j}{3}}^{\frac{8 \pi 2^{j}}{3}}\left(\frac{\partial H}{\partial \xi}\left(t^{\prime}, \xi\right) \xi^{-\left(\frac{1}{2}+H\left(t^{\prime}, \xi\right)\right)}-\frac{\partial H}{\partial \xi}(t, \xi) \xi^{-\left(\frac{1}{2}+H(t, \xi)\right)}\right) \ln (\xi) d \xi .
$$

The first term is split into

$$
\int_{\frac{2 \pi 2^{j}}{3}}^{\frac{8 \pi 2^{j}}{3}}\left(H\left(t^{\prime}, \xi\right)-H(t, \xi)\right) \xi^{-\left(\frac{3}{2}+H\left(t^{\prime}, \xi\right)\right)} d \xi
$$

which is of negligible compared to $J_{j}$, because of (13) for $k=1$ and into

$$
\int_{\frac{2 \pi 2^{j}}{3}}^{\frac{8 \pi 2^{j}}{3}} H(t, \xi)\left(\xi^{-\left(\frac{3}{2}+H\left(t^{\prime}, \xi\right)\right)}-\xi^{-\left(\frac{3}{2}+H(t, \xi)\right)}\right) d \xi
$$

which is also negligible compared to $J_{j, 0}$, because of (14) for $k=1$. With similar techniques it is shown that $J_{j, 2}$ is of the same order as $j J_{j, 0}$.

The contribution of the term $p=2$ in the sum (33) is estimated with similar arguments. Let us stress only one technical point. The partial derivative $\frac{\partial^{2}}{\partial \xi^{2}} \xi^{-\left(\frac{1}{2}+H(t, \xi)\right)}$ leads to consider a term :

$$
\int_{\frac{2 \pi 2 j}{3}}^{\frac{8 \pi 2^{j}}{3}}\left(\left|\frac{\partial H}{\partial \xi}\left(t^{\prime}, \xi\right)\right|^{2}\left(\xi^{-\left(\frac{1}{2}+H(t, \xi)\right)}-\xi^{-\left(\frac{1}{2}+H\left(t^{\prime}, \xi\right)\right)}\right)\right) \ln (\xi) d \xi .
$$

Because of (14) for $k=1$ we have

$$
\left|\frac{\partial H}{\partial \xi}\left(t^{\prime}, \xi\right)\right|^{2} \leq C \xi^{2 g-2}
$$

and we have seen that the preceding integral is conveniently bounded if $2 g<$ $a$. 


\subsection{Study of the Hölder regularity of the GMGP}

The Hölder regularity of the GMGP $X$, can be studied quite similarly to that of the GMBM [2] [4] and [5]. The proof of Theorem 1 follows exactly the same lines as that of Theorem 1.1 in [2], so we will only sketch it. Let $t_{0}$ be a fixed real, and let $Y_{t_{0}}$ be the Gaussian process defined for every real $t$ by

$$
Y_{t_{0}}(t)=\int_{0}^{+\infty} \frac{e^{-i t \cdot \xi}-1}{\xi^{1 / 2+H\left(t_{0}, \xi\right)}} W(d \xi)
$$

(this is nothing but $Y(s, t)$ where the second variable is fixed).

First step: Using (28), we show that almost surely,

$$
\alpha_{X}\left(t_{0}\right)=\alpha_{Y_{t_{0}}}\left(t_{0}\right)
$$

Second step: using (6), we show that for arbitrarily small $\epsilon>0$, some constant $\overline{c>0 \text { and every } t, t^{\prime}}$

$$
\mathbb{E}\left(\left|Y_{t_{0}}(t)-Y_{t_{0}}\left(t^{\prime}\right)\right|^{2}\right) \leq c\left|t-t^{\prime}\right|^{2\left(h\left(t_{0}\right)-\epsilon\right)} .
$$

Then a strong version of Kolmogorov criterion (see for instance Lemma 3.2 of [2]) implies that, almost surely, $\alpha_{Y_{t_{0}}}\left(t_{0}\right) \geq h\left(t_{0}\right)$.

Third step: using (6) again, we show that for every $t_{0}$ and arbitrarily small $\epsilon>0$

$$
\lim _{n \rightarrow \infty} \frac{\mathbb{E}\left(\left|Y_{t_{0}}\left(t_{0}+2^{-n}\right)-Y_{t_{0}}\left(t_{0}\right)\right|^{2}\right)}{2^{\left.-2 n\left(h\left(t_{0}\right)\right)+\epsilon\right)}}=+\infty,
$$

and then Lemma 2.2 of [2] implies that almost surely, $\alpha_{Y_{t_{0}}}\left(t_{0}\right) \leq h\left(t_{0}\right)$.

\subsection{Comparison of $W_{N}$ and $V_{N}$.}

In this section it is explained how estimates concerning $W_{N}$ can be transferred to $V_{N}$. We postpone the proof of

$$
\frac{1}{C} N^{1-2 b} \leq \frac{1}{C} N^{1-2 h_{*}} \leq W_{N} \leq C N^{1-2 h_{*}}
$$

to section 4.6. Let us now prove that if (37) is true we have

$$
\lim _{N \rightarrow+\infty} 1 / 2\left(1-\frac{\ln \left(V_{N}\right)}{\ln (N)}\right)=h_{*}
$$

which is the result we aim at. Let us rewrite (26) as

$$
W_{N}=\sum_{p=0}^{N-2}\left[\sum_{k=0}^{2} d_{k} Y\left(\frac{p+k}{N}, \frac{p}{N}\right)\right]^{2}
$$

where $d_{0}=1, d_{1}=-2$, and $d_{2}=1$; then $V_{N}$ can also be written: 


$$
V_{N}=\sum_{p=0}^{N-2}\left[\sum_{k=0}^{2} d_{k} Y\left(\frac{p+k}{N}, \frac{p+k}{N}\right)\right]^{2} .
$$

Hence we have

$$
\begin{aligned}
\left|W_{N}^{1 / 2}-V_{N}^{1 / 2}\right| & \leq\left(\sum_{p=0}^{N-2}\left[\sum_{k=0}^{2} d_{k}\left(Y\left(\frac{p+k}{N}, \frac{p}{N}\right)-Y\left(\frac{p+k}{N}, \frac{p+k}{N}\right)\right)\right]^{2}\right)^{1 / 2} \\
& \leq C\left(\sum_{p=0}^{N-2}\left[\left[\sum_{k=0}^{2}\left|\sup _{s \in[0,1]}\right| Y\left(s, \frac{p}{N}\right)-Y\left(s, \frac{p+k}{N}\right) \mid\right]^{2}\right)^{1 / 2}\right.
\end{aligned}
$$

and the following estimates are consequences of (28) and of (37):

$$
\begin{aligned}
\left|W_{N}^{1 / 2}-V_{N}^{1 / 2}\right| & \leq C N^{1 / 2-\beta} \\
\left|W_{N}^{1 / 2}-V_{N}^{1 / 2}\right| W_{N}^{-1 / 2} & \leq C N^{b-\beta} .
\end{aligned}
$$

Since $\beta>b$ it is clear by writing

$$
\ln \left(V_{N}^{1 / 2}\right)=\ln \left(1+\left(V_{N}^{1 / 2}-W_{N}^{1 / 2}\right) W_{N}^{-1 / 2}\right)+\ln \left(W_{N}^{1 / 2}\right)
$$

that

$$
\lim _{N \rightarrow+\infty} 1 / 2\left(1-\frac{\ln \left(V_{N}\right)}{\ln (N)}\right)=\lim _{N \rightarrow+\infty} 1 / 2\left(1-\frac{\ln \left(W_{N}\right)}{\ln (N)}\right)=h_{*}
$$

\subsection{Asymptotic of $\mathbb{E} W_{N}$}

In order to obtain the asymptotic of $W_{N}$, our next step is to study the asymptotic of $\mathbb{E} W_{N}$. Theorem 2 will then be a consequence of the asymptotic for the variance of $W_{N}$ and the Borel-Cantelli lemma. Let us rewrite $W_{N}$ as in (38) with definition (25):

$$
W_{N}=\sum_{p=0}^{N-2}\left(\int_{0}^{+\infty}\left(\sum_{k=0}^{2} d_{k} e^{-i \frac{p+k}{N} \xi}\right) \frac{W(d \xi)}{\xi^{H\left(\frac{p}{N}, \xi\right)+1 / 2}}\right)^{2}
$$

Because of the isometry given by the Wiener measure we have

$$
\mathbb{E} W_{N}=\sum_{p=0}^{N-2} \int_{0}^{+\infty} 16 \sin ^{4}\left(\frac{\xi}{2 N}\right) \frac{d \xi}{\xi^{2 H\left(\frac{p}{N}, \xi\right)+1}}
$$

Let us also remark that

$$
16 \int_{0}^{+\infty} \sin ^{4}\left(\frac{\xi}{2 N}\right) \frac{d \xi}{\xi^{2 h\left(\frac{p}{N}\right)+1}}=\frac{F\left(h\left(\frac{p}{N}\right)\right)}{N^{2 h\left(\frac{p}{N}\right)}}
$$


where

$$
F(s)=\int_{0}^{+\infty} 16 \frac{\sin ^{4}(\xi / 2)}{\xi^{2 s+1}} d \xi
$$

is a continuous function on the interval $(0,1)$ and hence is bounded on $[a, b]$.

Let us consider

$$
\widetilde{W}_{N}=\sum_{p=0}^{N-2} \frac{F\left(h\left(\frac{p}{N}\right)\right)}{N^{2 h\left(\frac{p}{N}\right)}}
$$

assumption A 1 yields

$$
\frac{1}{C} N^{1-2 h_{*}} \leq \widetilde{W}_{N} \leq C N^{1-2 h_{*}}
$$

Let us show that under assumption $\mathbf{A} \mathbf{2}$ we have

$$
\left|\mathbb{E} W_{N}-\widetilde{W}_{N}\right|=o\left(N^{1-2 h_{*}}\right)
$$

Because of (39) and (40)

$$
\left|\mathbb{E} W_{N}-\widetilde{W}_{N}\right| \leq \sum_{p=0}^{N-2} \int_{0}^{+\infty} 16 \sin ^{4}\left(\frac{\xi}{2 N}\right)\left|\xi^{-2 H\left(\frac{p}{N}, \xi\right)-1}-\xi^{-2 h\left(\frac{p}{N}\right)-1}\right| d \xi
$$

Then the integrals in (42) are split into three parts :

$$
\left|\mathbb{E} W_{N}-\widetilde{W}_{N}\right| \leq J_{N}^{1}+J_{N}^{2}+J_{N}^{3} .
$$

The first term is

$$
\begin{aligned}
J_{N}^{1} & =\sum_{p=0}^{N-2} \int_{0}^{1} 16 \sin ^{4}\left(\frac{\xi}{2 N}\right)\left|\xi^{-2 H\left(\frac{p}{N}, \xi\right)-1}-\xi^{-2 h\left(\frac{p}{N}\right)-1}\right| d \xi \\
& \leq C \sum_{p=0}^{N-2} \int_{0}^{1} \sin ^{4}\left(\frac{\xi}{2 N}\right) \frac{\left|H\left(\frac{p}{N}, \xi\right)-h\left(\frac{p}{N}\right)\right|}{\xi^{2 \theta\left(\frac{p}{N}, \xi\right)+1}} \ln (\xi) d \xi
\end{aligned}
$$

where $\theta\left(\frac{p}{N}, \xi\right) \in[a, b]$. Hence

$$
\begin{aligned}
J_{N}^{1} & \leq C \frac{N-1}{N^{4}} \int_{0}^{1} \xi \ln (\xi) d \xi \\
& \leq C / N^{3}
\end{aligned}
$$

and $J_{N}^{1}=o\left(N^{1-2 h_{*}}\right)$. Let us now consider

$$
\begin{aligned}
J_{N}^{2} & =\sum_{p=0}^{N-2} \int_{1}^{N^{\alpha}} 16 \sin ^{4}\left(\frac{\xi}{2 N}\right)\left|\xi^{-2 H\left(\frac{p}{N}, \xi\right)-1}-\xi^{-2 h\left(\frac{p}{N}\right)-1}\right| d \xi \\
& \leq C \sum_{p=0}^{N-2} \int_{1}^{N^{\alpha}} \sin ^{4}\left(\frac{\xi}{2 N}\right) \frac{\left|H\left(\frac{p}{N}, \xi\right)-h\left(\frac{p}{N}\right)\right|}{\xi^{2 \theta^{\prime}\left(\frac{p}{N}, \xi\right)+1}} \ln (\xi) d \xi
\end{aligned}
$$


where $\theta^{\prime}\left(\frac{p}{N}, \xi\right) \geq h\left(\frac{p}{N}\right)$. Hence

$$
\begin{aligned}
J_{N}^{2} & \leq \sum_{p=0}^{N-2} \frac{C}{N^{4}} \int_{1}^{N^{\alpha}} \frac{\xi^{3}\left|H\left(\frac{p}{N}, \xi\right)-h\left(\frac{p}{N}\right)\right|}{\xi^{2 h\left(\frac{p}{N}\right)}} \ln (\xi) d \xi \\
& \leq \sum_{p=0}^{N-2} \frac{C}{N^{4}} N^{\alpha\left(4-2 h\left(\frac{p}{N}\right)\right)} \ln (N) \\
& \leq C N^{4 \alpha-3-2 h_{*} \alpha} \ln (N)
\end{aligned}
$$

and since $\alpha<1, J_{N}^{2}=o\left(N^{1-2 h_{*}}\right)$. For the third part we have

$$
\begin{aligned}
J_{N}^{3} & =\sum_{p=0}^{N-2} \int_{N^{\alpha}}^{+\infty} 16 \sin ^{4}\left(\frac{\xi}{2 N}\right)\left|\xi^{-2 H\left(\frac{p}{N}, \xi\right)+1}-\xi^{-2 h\left(\frac{p}{N}\right)+1}\right| d \xi \\
& \leq C \sum_{p=0}^{N-2} \int_{N^{\alpha}}^{+\infty} \frac{\left|H\left(\frac{p}{N}, \xi\right)-h\left(\frac{p}{N}\right)\right|}{\xi^{2 \theta^{\prime \prime}\left(\frac{p}{N}, \xi\right)+1}} \ln (\xi) d \xi
\end{aligned}
$$

where $\theta^{\prime \prime}\left(\frac{p}{N}, \xi\right) \geq h\left(\frac{p}{N}\right)$.

$$
J_{N}^{3} \leq C \sum_{p=0}^{N-2} \int_{N^{\alpha}}^{+\infty} \frac{\left|H\left(\frac{p}{N}, \xi\right)-h\left(\frac{p}{N}\right)\right|}{\xi^{2 h\left(\frac{p}{N}\right)+1}} \ln (\xi) d \xi
$$

and $J_{N}^{3}=o\left(N^{1-2 h_{*}}\right)$ because of A 2. Hence

$$
\left|\mathbb{E} W_{N}-\widetilde{W}_{N}\right|=o\left(N^{1-2 h_{*}}\right)
$$

and

$$
\frac{1}{C} N^{1-2 h_{*}} \leq \mathbb{E} W_{N} \leq C N^{1-2 h_{*}}
$$

is obtained.

\subsection{Asymptotic of $\operatorname{var}\left(W_{N}\right)$}

Let us now show that almost surely

$$
\lim _{N \rightarrow+\infty} \frac{W_{N}}{\mathbb{E} W_{N}}=1 .
$$

Since the process $X$ is Gaussian, classical formulas for the variance of Gaussian variables leads to :

$$
\begin{aligned}
\operatorname{var}\left(W_{N}\right)=2 \sum_{p, p^{\prime}=0}^{N-2} \mathbb{E}\left(\int_{0}^{+\infty}\left(\sum_{k=0}^{2} d_{k} e^{-i \frac{p+k}{N} \xi}\right) \frac{W(d \xi)}{\xi^{H\left(\frac{p}{N}, \xi\right)+1 / 2}}\right. \\
\left.\int_{0}^{+\infty}\left(\sum_{k=0}^{2} d_{k} e^{i \frac{p^{\prime}+k}{N} \xi}\right) \frac{W(d \xi)}{\xi^{H\left(\frac{p^{\prime}}{N}, \xi\right)+1 / 2}}\right)^{2}
\end{aligned}
$$




$$
\begin{aligned}
\operatorname{var}\left(W_{N}\right)=2 \sum_{p, p^{\prime}=0}^{N-2}\left(\int_{0}^{+\infty} e^{i\left(\frac{p^{\prime}-p}{N}\right) \xi} 16 \sin ^{4}\right. & \left(\frac{\xi}{2 N}\right) \\
& \left.\frac{d \xi}{\xi^{H\left(\frac{p}{N}, \xi\right)+H\left(\frac{p^{\prime}}{N}, \xi\right)+1}} d \xi\right)^{2} .
\end{aligned}
$$

At this point one needs similar estimates for the integrals in the previous formula than the one we had when we considered $\mathbb{E} W_{N}$. However to apply the Borel-Cantelli Lemma we aim at

$$
\operatorname{var}\left(W_{N}\right)=O\left(N^{1+2 g-4 h_{*}} \ln ^{2}(N)\right)
$$

and we prove

$\left|\int_{0}^{+\infty} e^{i\left(\frac{p^{\prime}-p}{N}\right) \xi} 16 \sin ^{4}\left(\frac{\xi}{2 N}\right) \frac{d \xi}{\xi^{H\left(\frac{p}{N}, \xi\right)+H\left(\frac{p^{\prime}}{N}, \xi\right)+1}} d \xi\right| \leq C \frac{N^{g} \ln (N)}{N^{h\left(\frac{p}{N}\right)+h\left(\frac{p^{\prime}}{N}\right)}} \frac{1}{\left|p-p^{\prime}\right|+1}$

which yields the needed estimates. Once more the factor $\frac{1}{\left|p-p^{\prime}\right|+1}$ that allows us to have a better estimate for $g<1 / 2$ than $\operatorname{var}\left(W_{N}\right)=O\left(N^{2-4 h_{*}} \ln ^{2}(N)\right)$ is obtained by integrations by parts in the integrals of (45). Let us compute

$$
\begin{aligned}
& \frac{\partial}{\partial \xi}\left(\frac{\sin ^{4}\left(\frac{\xi}{2 N}\right)}{\left.\xi^{H\left(\frac{p}{N}, \xi\right)+H\left(\frac{p^{\prime}}{N}, \xi\right)+1}\right)}\right) \\
& \frac{2 \sin ^{3}\left(\frac{\xi}{2 N}\right) \cos \left(\frac{\xi}{2 N}\right)}{N \xi^{H\left(\frac{p}{N}, \xi\right)+H\left(\frac{p^{\prime}}{N}, \xi\right)+1}}-\frac{\sin ^{4}\left(\frac{\xi}{2 N}\right)\left(H\left(\frac{p}{N}, \xi\right)+H\left(\frac{p^{\prime}}{N}, \xi\right)+1\right)}{\xi^{H\left(\frac{p}{N}, \xi\right)+H\left(\frac{p^{\prime}}{N}, \xi\right)+2}} \\
& \\
&-\frac{\sin ^{4}\left(\frac{\xi}{2 N}\right)\left(\frac{\partial H}{\partial \xi}\left(\frac{p}{N}, \xi\right)+\frac{\partial H}{\partial \xi}\left(\frac{p^{\prime}}{N}, \xi\right)\right) \ln (\xi)}{\xi^{H\left(\frac{p}{N}, \xi\right)+H\left(\frac{p^{\prime}}{N}, \xi\right)+1}} .
\end{aligned}
$$

Then the contribution of the first term of (46) to (45) is given by

$$
4 \int_{0}^{+\infty} \frac{e^{i\left(\frac{\left(p^{\prime}-p\right) \xi}{N}\right)} \sin ^{3}\left(\frac{\xi}{2 N}\right) \cos \left(\frac{\xi}{2 N}\right) d \xi}{i\left(p-p^{\prime}\right) \xi^{H\left(\frac{p}{N}, \xi\right)+H\left(\frac{p^{\prime}}{N}, \xi\right)+1}}
$$

when $p-p^{\prime} \neq 0$. This integral is then easily bounded by :

$$
I_{1}=\frac{4}{\left|p-p^{\prime}\right|} \int_{0}^{+\infty} \frac{\left|\sin ^{3}\left(\frac{\xi}{2 N}\right)\right| d \xi}{\xi^{H\left(\frac{p}{N}, \xi\right)+H\left(\frac{p^{\prime}}{N}, \xi\right)+1}}
$$

which we split into three terms to get the required estimates. The first one is :

$$
\begin{aligned}
I_{1,1} & =\frac{4}{\left|p-p^{\prime}\right|} \int_{0}^{1} \frac{\left|\sin ^{3}\left(\frac{\xi}{2 N}\right)\right| d \xi}{\xi^{H\left(\frac{p}{N}, \xi\right)+H\left(\frac{p^{\prime}}{N}, \xi\right)+1}} \\
& \leq \frac{C}{N^{3}\left|p-p^{\prime}\right|} ;
\end{aligned}
$$


the second one is

$$
\begin{aligned}
I_{1,2} & =\frac{4}{\left|p-p^{\prime}\right|} \int_{1}^{N} \frac{\left|\sin ^{3}\left(\frac{\xi}{2 N}\right)\right| d \xi}{\xi^{H\left(\frac{p}{N}, \xi\right)+H\left(\frac{p^{\prime}}{N}, \xi\right)+1}} \\
& \leq \frac{C}{N^{3}\left|p-p^{\prime}\right|} \int_{1}^{N} \xi^{2-h\left(\frac{p}{N}\right)-h\left(\frac{p^{\prime}}{N}\right)} d \xi \\
& \leq \frac{C}{N^{h\left(\frac{p}{N}\right)+h\left(\frac{p^{\prime}}{N}\right)}\left|p-p^{\prime}\right|}
\end{aligned}
$$

and the third one

$$
\begin{aligned}
I_{1,3} & =\frac{4}{\left|p-p^{\prime}\right|} \int_{N}^{+\infty} \frac{\left|\sin ^{3}\left(\frac{\xi}{2 N}\right)\right| d \xi}{\xi^{H\left(\frac{p}{N}, \xi\right)+H\left(\frac{p^{\prime}}{N}, \xi\right)+1}} \\
& \leq \frac{C}{\left(N^{h\left(\frac{p}{N}\right)+h\left(\frac{p^{\prime}}{N}\right)}\right)\left(\left|p-p^{\prime}\right|\right)}
\end{aligned}
$$

Hence

$$
\left|I_{1}\right| \leq\left(\frac{C_{1}}{N^{3}}+\frac{C_{2}}{N^{h\left(\frac{p}{N}\right)+h\left(\frac{p^{\prime}}{N}\right)}}\right)\left(\frac{1}{\left|p-p^{\prime}\right|+1}\right)
$$

where the easier case $p=p^{\prime}$ is also considered. With similar arguments we get for the second term of (46)

$$
\left|I_{2}\right| \leq\left(\frac{C_{1}}{N^{3}}+\frac{C_{2}}{N^{h\left(\frac{p}{N}\right)+h\left(\frac{p^{\prime}}{N}\right)}}\right)\left(\frac{N^{g}}{\left|p-p^{\prime}\right|+1}\right)
$$

and the last term of (46) yields

$$
\left|I_{3}\right| \leq\left(\frac{C_{3}}{N^{3}}+\frac{C_{4}}{N^{h\left(\frac{p}{N}\right)+h\left(\frac{p^{\prime}}{N}\right)}}\right)\left(\frac{N^{g} \ln (N)}{\left|p-p^{\prime}\right|+1}\right)
$$

where (14) has been used for $k=1$. Hence we deduce that

$$
\operatorname{var}\left(W_{N}\right)=O\left(N^{1+2 g-4 h_{*}} \ln ^{2}(N)\right) .
$$

\subsection{Almost sure convergence}

In order to show that

$$
\lim _{N \rightarrow+\infty} \frac{W_{N}}{\mathbb{E} W_{N}}=1 \quad \text { almost surely }
$$

we apply Markov inequality for $A>0$ :

$$
\mathbf{P}\left(\left|\frac{W_{N}}{\mathbb{E} W_{N}}-1\right|^{4} \geq A\right) \leq \mathbb{E}\left|\frac{W_{N}}{\mathbb{E} W_{N}}-1\right|^{4} / A
$$


Moreover the same arguments as in the proof of Proposition 2 p. 43 of [9] yield that

$$
\mathbb{E}\left|W_{N}-\mathbb{E} W_{N}\right|^{4} \leq C \operatorname{var}\left(W_{N}\right)^{2}
$$

then

$$
\mathbf{P}\left(\left|\frac{W_{N}}{\mathbb{E} W_{N}}-1\right|^{4} \geq A\right) \leq \frac{C \operatorname{var}\left(W_{N}\right)^{2}}{\left(\mathbb{E} W_{N}\right)^{4}}
$$

For $g<1 / 4$ the sequence

$$
\frac{\operatorname{var}\left(W_{N}\right)^{2}}{\left(\mathbb{E} W_{N}\right)^{4}} \leq C N^{4 g-2} \ln ^{2}(N)
$$

is summable and the Borel-Cantelli lemma yields the required almost sure convergence. With (43) and the results of section 4.3, this fact concludes the proof of Theorem 2.

\subsection{Proof of Corollary 1}

To prove the corollary, let us fix $t \in(0,1)$ and set

$$
h_{*}(\varepsilon)=\inf _{\{|s-t|<\varepsilon\}} h(s)
$$

Let also

$$
\mathbf{W}_{\varepsilon, N}=\sum_{p \in \mathcal{V}_{\varepsilon, N}(t)}\left(Y\left(\frac{p+2}{N}, \frac{p}{N}\right)-2 Y\left(\frac{p+1}{N}, \frac{p}{N}\right)+Y\left(\frac{p}{N}, \frac{p}{N}\right)\right)^{2} .
$$

With the same arguments as in the proof of Theorem 2

$$
\frac{\varepsilon}{C} N^{1-2 h_{*}(\varepsilon)} \leq \mathbb{E} \mathbf{W}_{\varepsilon, N} \leq C \varepsilon N^{1-2 h_{*}(\varepsilon)}
$$

and, if we take $\varepsilon=N^{-\gamma}$,

$$
\lim _{N \rightarrow+\infty} 1 / 2\left(1-\frac{\ln \left(\mathbb{E} \mathbf{W}_{N^{-\gamma}, N}\right)}{\ln N}\right)=\liminf _{s \rightarrow t} h(s) .
$$

Moreover

$$
\operatorname{var}\left(\mathbf{W}_{\varepsilon, N}\right)=O\left(\varepsilon N^{1+2 g-4 h_{*}(\varepsilon)} \ln ^{2}(N)\right)
$$

and if we take $0<\gamma<1 / 2$,

$$
\lim _{N \rightarrow+\infty} \frac{\mathbf{W}_{N^{-\gamma}, N}}{\mathbb{E} \mathbf{W}_{N^{-\gamma}, N}}=1
$$

almost surely. Next

$$
\left|\mathbf{W}_{\varepsilon, N}^{1 / 2}-\mathbf{W}_{\varepsilon, N}^{1 / 2}\right| \mathbf{W}_{\varepsilon, N}^{-1 / 2} \leq C N^{b-\beta}
$$

is still true and the corollary is proved. 


\section{References}

1. Ayache, A.: 2001, Du mouvement Brownien fractionnaire au mouvement Brownien multifractionnaire, Technique et science informatiques, 20, 9 11331152.

2. Ayache, A.: 2000, The generalized multifractional Brownian motion can be multifractal., Technical Report LSP-2000-22, Laboratoire de Statistique et Probabilités, UMR C5583 Université Paul Sabatier.

3. Ayache, A. and Lévy Véhel, J.: 2001, Processus à régularité locale prescrite, Comptes Rendus Académie des Sciences de Paris 333, I, 233-238.

4. Ayache, A. and Lévy Véhel, J.: 2000, The Generalized Multifractional Brownian Motion. Statistical Inference for Stochastic Processes 3, 7-18.

5. Ayache, A. and Lévy Véhel, J.: 1999, Generalized multifractional Brownian motion: Definition and preliminary results, in M. Dekking, J. Lévy Véhel, E. Lutton and C. Tricot (eds), Fractals : Theory and Applications in Engineering, Springer Verlag, 17-32.

6. Beran, J. : Statistics for Long-Memory Processes, Chapman and Hall, London, U.K., 1994.

7. Benassi, A., Bertrand, P., Cohen, S. and Istas, J.: 2000, Identification of the Hurst exponent of a step multifractional Brownian motion, Statistical Inference for Stochastic Processes 3, 101-110.

8. Benassi, A., Cohen, S. and Istas, J.: 1998a, Identifying the multifractional function of a Gaussian process, Statistic and Probability Letters 39, 337-345.

9. Benassi, A., Cohen, S., Istas, J. and Jaffard, S.: 1998b, Identification of Filtered White Noises, Stoch. Proc. Appl. 75, 31-49.

10. Benassi, A., Jaffard, S. and Roux, D.: 1997, Gaussian processes and Pseudodifferential Elliptic operators, Revista Mathematica Iberoamericana 13(1), 19-89.

11. Canus, C. and Lévy Véhel, J.: 1996, Change detection in sequences of images by multifractal analysis, Proc. ICASSP-96, May 7-10, Atlanta, GA.

12. Cohen, S.: 1999, From self-similarity to local self-similarity : the estimation problem, in M. Dekking, J. Lévy Véhel, E. Lutton and C. Tricot (eds), Fractals : Theory and Applications in Engineering, Springer Verlag, pp. 3-16.

13. Cohen, S.: 2001, Champs localement auto-similaires, in P. Abry, P. Gonçalvès and J. Lévy Véhel (eds), Fractal et Lois d'échelles, Hermes.

14. Daoudi, K., Lévy Véhel, J. and Meyer, Y.:1996, Construction of continuous functions with prescribed local regularity, Const. Approx. 014, (03) 349-385.

15. Frisch, U. and Parisi, G.: 1985, Turbulence and predictability in geophysical fluid dynamics and climate dynamics, Ghil,M. Benzi, R. and Parisi, G. eds, Amsterdam (Holland).

16. Kolmogorov, A.: 1940, Wienersche Spiralen und einige andere interessante Kurven in Hilbertsche Raum., C. R. (Dokl.) Acad. Sci. URSS 26, 115-118.

17. Lévy Véhel, J.: 1996, Introduction to the multifractal analysis of images, in Fractal Geometry and Analysis, The Mandelbrot Festschrift, Curacao 1995, C.J.G. Evertsz, H.-O. Peitgen and R.F. Voss Editors, World Scientific,1996.

18. Lévy Véhel, J. and Mignot, P.: 1994, Multifractal Segmentation of Images, Fractals 2(3), 371-378.

19. Mandelbrot, B. and Van Ness, J.: 1968, Fractional Brownian motion, fractional noises and applications, Siam Review 10, 422-437.

20. Meyer, Y.: 1990, Ondelettes et Opérateurs, Vol. 1, Hermann, Paris. 
21. Ndoye, M.: 2001, Estimation de la fonction d'identification d'un processus gaussien multifractionnaire : le cas Hölderien, private communication.

22. Peltier, R. and Lévy Véhel, J.: 1996, Multifractional Brownian motion: definition and preliminary results, available at http://www-rocq.inria.fr/fractales/. 